\title{
Was dämmt die Gewalt in der Pflege?
}

\begin{abstract}
Ausgangslage: Die Studienautoren stellten fest, dass es keine Hinweise für einen Rückgang von Gewalt in der Pflege gibt, obwohl wissenschaftliche Erkenntnisse den Weg zu einer Eindämmung der Gewalt von Pflegenden gegenüber Pflegebedürftigen aufzeigen. Beispielsweise ist bekannt, dass auch Faktoren wie die mangelnde berufliche Qualifikation Gewalt in der Pflege bewirken. Die Wissenschaftlerinnen untersuchten daraufhin, warum der Transfer dieser Erkenntnisse in den Pflegealltag nicht gelingt.
\end{abstract}

» In dieser nicht repräsentativen Studie wurden 20 Personen aus neun stationären und einer ambulanten Einrichtung aus dem Raum Düsseldorf befragt; davon sind zehn Personen in der Pflege und zehn Personen in der Leitung tätig. Zwei separate Interviewleitfäden kamen zum Einsatz, einer für die Pflegenden und einer für die Leitungskräfte. Die Studie identifiziert folgende Ursachen für Gewalt in der Pflege:

1. Pflegende sind sich unsicher, wie sie Kollegen auf bei ihnen beobachtete Gewalt ansprechen können.

2. Entweder haben die Einrichtungen keine Standards zum Umgang mit problematischen Situationen oder die Pflegenden kennen den Inhalt solcher Standards nicht. Dies führe wiederum dazu, dass Gewalt nicht ausreichend dokumentiert werde, obwohl man dadurch Risiken und Probleme rechtzeitig erkennen könne. Dies wird auf die eher allgemein gewonnene Erkenntnis zurückgeführt, dass den Pflegenden die Bedeutung der Dokumentation nicht bewusst ist und sie diese für lästige Bürokratie hielten.
3. Die Rahmenbedingungen der Pflege - Personalmangel, Zeitdruck, ungleiches Machtgefüge und lange Arbeitsperioden - begünstigen Gewalt.

4. Aggressives oder störendes Verhalten der Pflegebedürftigen provoziert Gewalt der Pflegenden.

5. Überforderung, geringe Qualifikation, eigene Gewalterfahrungen der Pflegenden und „mangelnde Identifikation mit dem Beruf Altenpflege" fördern die Anwendung von Gewalt. Die Arbeitsagentur würde aus arbeitsmarktpolitischen Erwägungen ungeeignete Personen in das „beschäftigungspolitische Auffangbecken" der Pflege schicken.

6. Es gibt zu wenig Fortbildungen zur Gewaltprävention, obwohl das Wohnund Teilhabegesetz von 2014 die Einrichtungen zu Maßnahmen zur Gewaltprävention verpflichtet und in einer Interventionsstudie nachgewiesen wurde, dass Gruppenschulungen zum Thema Gewalt in der Folge die psychische Gewalt reduzierte.

7. Gewalt gegenüber Pflegebedürftigen wird von der Leitung vertuscht,

\section{Kommentar}

Konsens ist wohl, dass Gewalt in der Pflege unbedingt verhindert werden muss. Bei dieser explorativen Studie bewegen sich die Gerichtsmedizinerinnen auf ihnen bisher unbekanntes Terrain vor. Wenn man aber, anstatt im Feld Beobachtungen anzustellen, Personen aus dem Feld befragt, erhält man vor allem Meinungen, aber selten Fakten; da kann man schon mal von den befragten Personen aufs Glatteis geführt werden. Beispiele: 1. Gewalt in der Pflege ginge vor allem von Pflegehilfskräften, die angeblich gegen ihren Willen von der Arbeitsagentur zur Altenpflege verpflichtet wurden, aus. Eine unbewiesene Behauptung, die aber von den Forscherinnen ungeprüft in den Katalog der Gewaltursachen mit aufgenommen wird. 2. Die gut reflektierte Kritik zahlloser Pflegender an der überbordenden Dokumentation basiert nicht auf Ignoranz, sondern auf jahrzehntelangen Erfahrungen mit sinnlosen Formulareinträgen, die sich nie wieder jemand ansieht. Und es ist auch nicht einzusehen, warum weniger Gewalt in der Pflege ausgeübt wird, wenn Pflegende mehr darüber schreiben. weil jene eine negative Auswirkung auf die Öffentlichkeit scheuen.

8. Es läge keine allgemein verbindliche Definition des Gewaltbegriffs vor und die Pflegenden wissen gar nicht, wo und wann Gewalt beginne.

\section{Empfohlene Maßnahmen gegen Gewalt}

Als Maßnahmen gegen die Gewalt empfehlen die Studienautoren daher die Formulierung eines gemeinsamen Gewaltbegriffs. Die Einrichtungen sollen Standards zum Umgang mit schwierigen Situationen erstellen. Und es müsse in den Pflegeeinrichtungen eine „Kultur der Offenheit“ entwickelt werden, in der man sich angstfrei zu eigenen aggressiven Handlungen bekennen könne. Eine klare Präventionsstrategie benennt zudem Verantwortlichkeiten und Ansprechpartner, die öffentlich bekannt gemacht werden sollen. Außerdem sollten Fortbildungen zum Thema Gewalt durchgeführt werden und die Personalauswahl sorgfältiger erfolgen. Man solle nur jene Personen einstellen, die über ein entsprechendes Sozialverhalten, kommunikative Kompetenz und Sensibilität verfügen; die Lücken im Dienstplan sollten nicht so oft durch Aushilfskräfte geschlossen werden.

Quelle:

M. Siegel, Y. Mazheika, R. Mennicken; S. Ritz-Timme, H. Graß, B. Gahr, „Weil wir spüren, da müssen wir was tun"-Barrieren in der Gewaltprävention sowie zentrale Handlungserfordernisse. Z Gerontol Geriat DOI 10.1007/s00391-017-1228-0

Autorenkontakt:

Referiert und kommentiert von Markus Hieber, Diplom-Pflegewirt (FH), M.A., Altenpfleger Berlin E-Mail: markus_hieber@t-online. de 